\title{
Electrocutaneous spatial integration at threshold: The effects of electrode size
}

\author{
ATSUKI HIGASHIYAMA \\ University of Osaka Prefecture, Osaka, Japan \\ and \\ TAKARA TASHIRO \\ Osaka City University, Osaka, Japan
}

\begin{abstract}
Five experiments were carried out to investigate the effects of electrode size on threshold for current pulses that flowed between the ventral and dorsal sides of the right forearm. The main result was that the total current threshold decreases as the size of the cathode and anode increases at least up to $15 \mathrm{~mm}$ in diameter. To account for this finding, a neural summation model was proposed, assuming that the central nervous system sums up neural impulses discharged near the conduction paths under electrodes. It was also found that the current flowing from the dorsal to the ventral side provided lower thresholds and more stable localization than the opposite flow of current did. This finding suggested that current threshold and its perceived locus are determined by both the body site on which electrodes were placed and the relative polarity that was assigned to electrodes.
\end{abstract}

Our purpose in this paper is to discuss the effects of electrode size on electrocutaneous threshold. Although there are a variety of possible electrode configurations used in electrocutaneous stimulation (e.g., Sherrick \& Cholewiak, 1986), let us consider a simple case in which an electrode is placed on the ventral side of the forearm and another electrode on its dorsal side.

One prediction regarding the effects of electrode size on threshold would follow from the knowledge of spatial summation that is commonly found in other cutaneous sensations such as vibrotaction (e.g., Verrillo, 1963), or warmth and cold (e.g., Kenshalo, 1970). Spatial summation refers to the dependence of threshold and of sensory magnitude on the areal size of a stimulus (Marks, 1974). For example, the vibrotactile threshold for the hand decreases by $3 \mathrm{~dB}$ for every doubling of contact area (Verrillo, 1963). Similarly, the warmth threshold also decreases by a half for every doubling of the skin area exposed by radiant heat (Kenshalo, 1970). Thus, complete summation-the precise reciprocal relation between threshold (or sensory magnitude) and stimulus area-holds for vibrotactile and warmth thresholds.

It is therefore very likely that spatial summation takes place for electrocutaneous stimulation as well. If thresholds are measured in terms of current density (i.e., the amount of current flow per unit area), it can be predicted that, as the contact area of electrodes increases, the threshold will decrease until the critical area is exceeded and that then it will remain constant.

Correspondence should be addressed to Atsuki Higashiyama, Psychology Laboratory, University of Osaka Prefecture, Sakai, Osaka 591, Japan.
However, it is possible to measure thresholds in terms of the total current flowing between electrodes. In this case, the thresholds for perfect summation would remain constant regardless of contact area, whereas the thresholds for partial or no summation would increase with increasing contact area.

In this study, thresholds were measured in terms of total current, rather than current density, because it is difficult to keep current density constant at all tissues intervening between electrodes. That is, current density is generally high beneath the electrodes, but it is low at the midpoint between the electrodes (Békésy, 1959b, Figure 15; Benton, Baker, Bowman, \& Waters, 1981; Ranck, 1981).

Some research done with two pairs of electrodes may have suggested spatial summation for electrical stimulation. Vernon and Wessman (1956) placed four electrodes along the palmar surface of the middle finger, and delivered sinusoidal current so that the two outside electrodes completed one electrical circuit and the two inside electrodes completed another one. When the current that was $75 \%$ of the threshold flowed through the outside pair, the threshold for the inside pair varied as a function of the phase relation between the two currents. In particular, the threshold for the in-phase relation was lower than that for the out-of-phase relation, suggesting summation of current for the in-phase relation. Békésy (1959a) measured the sensation magnitude that was produced by two pairs of concentric electrodes on the palm of the hand. When the distance between the electrode pairs was so small as to fuse the two shocks, the fused sensation was greater than the sensation produced by each pair alone.

A different prediction has been suggested by Benton et al. (1981, chap. 3) and McNeal (1973), who assumed 
that the apparent magnitude of electrocutaneous stimulation is determined by the current density, rather than the total current between electrodes. They assumed that electrical stimulation tends to depolarize nerves under the cathode and that, when the total current between electrodes is constant, a great sensation will be yielded under a small cathode, which gives rise to high current density. This assumption can lead us to the prediction that the total current required to attain threshold sensation increases as the cathode area increases. We refer to this prediction as the density hypothesis. The density hypothesis can be supported through reference to Green (1962), who found that when a current of $1-\mathrm{sec}$ duration flowed between the thumb and index finger of the left hand, the thresholds for the electrodes $1.9,3.8$, and $7.6 \mathrm{~mm}$ in diameter were 225,313 , and $458 \mu \mathrm{A}$, respectively.

Five experiments were performed to examine the relative effectiveness of cathode and anode sizes on thresholds for single current pulses. In all but Experiment 4, the cathode was positioned on the inner or ventral side of the right forearm and the anode on its outer or dorsal side. In Experiment 1, a threshold for pulses of 2-msec duration was determined for each combination of two cathode sizes and three anode sizes. From the summation hypothesis, it can be predicted that thresholds will remain constant independently of the cathode size (perfect summation), or that higher threshold will be obtained for larger cathode size (partial or no summation), whereas from the density hypothesis, it can be predicted that higher thresholds will be obtained for larger cathode size. In either hypothesis, the role of the anode with respect to threshold is not clear, because it is assumed that threshold sensation does not take place under the anode (Benton et al., 1981).

Experiment 2 was a parametric one in which the effects of cathode size were explored for a given size of the anode. Experiment 3 was also a parametric one as to the effects of anode size, for a given size of the cathode.

In Experiment 4, the interaction between the effects of electrode size on threshold and the flow of current was examined. In this experiment, the thresholds for current flowing from the dorsal to the ventral side of the forearm were compared with those for the opposite flow of current. For each flow of current, a constant electrode was placed on the dorsal or ventral side, and a variable electrode was placed on the opposite side.

In Experiment 5, the interaction of electrode size and pulse duration was examined. The current threshold was determined for pulses of 1-, 10-, and 100-msec durations, presented with two different cathode sizes.

\section{GENERAL METHOD}

\section{Apparatus}

A Nihonkoden SEN-7203 electric stimulator provided the basic pulse-forming and -timing circuit. It was used with a Nihonkoden SS-102J isolator, which, operating at $200 \mathrm{~V}$, amplified the basic current pulse delivered by the stimulator and provided a pulse of controllable amplitude by adjusting a variable resistor in the stimulator.
A system consisting of an Apple II microcomputer and a Sanwa time regulator determined the time schedule of the warning buzzer, the foreperiod, and the intertrial interval. Pulse shape and duration were calibrated by a Hitachi Type V-302 oscilloscope; pulse amplitude was measured by a Sanwa Electronic Instrument Model $9000 \mathrm{EA}$ digital ammeter. Higashiyama and Tashiro (1988) have provided more details of the apparatus.

The use of the constant-current stimulator was due to a desire to compensate for instabilities in skin impedance. In our stimulator, the total number of electrons flowing between electrodes was designed to remain constant whenever the product of the current, $I$, and the skin impedance, $R$, was below $200 \mathrm{~V}(I R<200)$. Since the current used in this study ranged from 0.3 to $5.0 \mathrm{~mA}$, this stimulator guaranteed constant current only for skin impedances of $40 \mathrm{k} \Omega$ or less.

It appears that skin impedance at threshold varies from 5 to $30 \mathrm{k} \Omega$, depending on physical (e.g., current amplitude, electrode size, pulse width, pulse repetition rate, and trial number) and physiological (e.g., skin treatment, body site, and age) parameters. Saunders (1973, Figure 5), for example, found that the skin impedances for a $40-\mathrm{Hz}$ pulse train were 37,18 , and $7 \mathrm{k} \Omega$ for active electrodes 1,2 , and $3 \mathrm{~mm}$ in diameter, respectively. Tursky and Watson (1964) also found that although skin impedance varies with electrode size, shape, interelectrode distance, and sensation level, it generally hovers around about $20 \mathrm{k} \Omega$ at threshold. Thus, our stimulator seems to be qualified as a constant-current stimulator.

\section{Electrodes}

The electrodes were flat silver disks filled with keratin electrode paste and fastened to the skin with 3M Transpore No. 1527 surgical tape. An electrode was positioned on the ventral side of the right forearm and another one on its dorsal side. In Experiments 1, 2, 3 , and 5, the cathode was placed on the ventral side and the anode on the dorsal side. Thus, current flowed from the dorsal to the ventral. In Experiment 4, current flowed in two directions-from the dorsal to the ventral, and from the ventral to the dorsal; the polarity of the electrodes was reversed.

\section{Procedure}

Each subject was seated, with the right hand resting on the table. A pair of electrodes was attached to the subject's forearm after washing it with an alcohol solution.

An absolute threshold for single pulses was determined with a staircase procedure for each pair of electrodes. The subject was asked to make more than 32 "yes"/ "no" judgments of whether or not current was present on the skin. If the current was detected, it was decreased by a step (about $.08 \mathrm{~mA}$ ); if not, it was increased by a step. A current pulse was presented on every trial; there were no blank trials. After completing the judgments for each pair of electrodes, the subject was asked to point to the perceived locus of the electrical pulses. When current was perceived under the ventral side, the subjects reported "inside," and when it was perceived under the dorsal side, they reported "outside." When the subjects perceived current under both electrodes, they reported "both."

Each trial sequence was started with a warning buzzer; this was followed by a pulse to the electrodes after a foreperiod of 2.16 , 3.47 , or $4.81 \mathrm{sec}$. The foreperiod was randomized for each trial. The intertrial interval was about $10 \mathrm{sec}$. When the subject reported "yes" during the foreperiod (i.e., a false alarm), the experimenter provided verbal feedback, and the subject was asked to avoid such a response by changing the judgmental criterion.

In Experiments 1, 2, 3, and 5, there were six different pairs of electrodes, and in Experiment 4, there were 12 different pairs of electrodes. These pairs of electrodes were presented one at a time. The presentation order of electrode pairs was different for each subject. It took about $8 \mathrm{~min}$ to determine a threshold for an electrode pair. 


\section{EXPERIMENT 1}

\section{Method}

A threshold for pulses of 2-msec duration was determined for each combination of two cathode sizes $(2.5$ and $30 \mathrm{~mm}$ in diameter) and three anode sizes $(2.5,15$, and $30 \mathrm{~mm}$ in diameter). Fourteen undergraduates served as the subjects; they were paid for their participation.

\section{Results and Discussion}

For every pair of electrodes, all subjects perceived cutaneous sensations under the cathode on the ventral side of forearm. They did not report sensations under the anode on the dorsal side.

The thresholds obtained are shown in Figure 1, where mean threshold taken across the subjects is plotted against contact area of cathode, with anode size as the parameter.

A two-way analysis of variance for repeated measures was performed on the threshold data. The results showed that the main effect of cathode was significant $[F(1,13)=$ $25.4, p<.001]$, indicating that threshold was higher for the $2.5-\mathrm{mm}\left(4.9-\mathrm{mm}^{2}\right)$ cathode than for the $30-\mathrm{mm}$ $\left(706.5-\mathrm{mm}^{2}\right)$ cathode. The main effect of anode was also significant $[F(2,26)=52.2, p<.001]$. The cathode $\times$ anode interaction was not significant $(F<1)$. Contrast analyses (Rosenthal \& Rosnow, 1985) showed that the 2.5-mm anode produced significantly higher thresholds than did the 15 -mm anode $[F(1,26)=69.4, p<.001]$ and the 30 -mm anode $[F(1,26)=85.9, p<.001]$, but that the threshold for the $15-\mathrm{mm}$ anode was not significantly different from that for the 30 -mm anode $(F<1)$.

It was thus shown that (1) the highest threshold was obtained when both electrodes were small, (2) the lowest threshold was obtained when both electrodes were large, and (3) the intermediate thresholds were obtained when one electrode was large and the other was small. It is of interest that threshold varied as a function of not only cathode size but also anode size, although current was per-

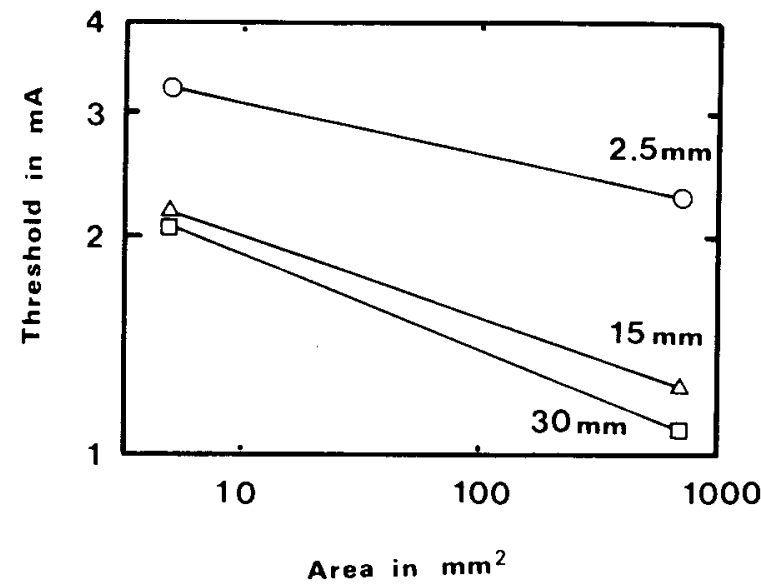

Figure 1. Threshold (in milliamperes) as a function of cathode area (in square millimeters). Current flowed from the dorsal (anode) to the ventral (cathode). The parameter is the anode diameter: circles, $2.5 \mathrm{~mm}$; triangles, $15 \mathrm{~mm}$; and squares, $30 \mathrm{~mm}$.

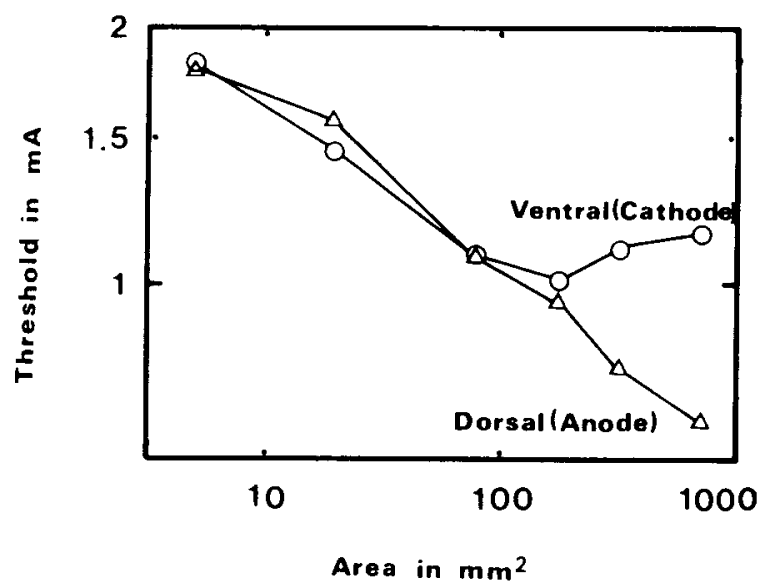

Figure 2. Threshold (in milliamperes) as a function of area (in square millimeters) of variable electrode. Current fowed from the dorsal (anode) to the ventral (cathode). The parameter is the site (polarity) of the variable electrode: circles, ventral (cathode); triangles, dorsal (anode).

ceived only under the cathode, which was always on the ventral side.

\section{EXPERIMENTS 2 AND 3}

Our purpose in Experiments 2 and 3 was to examine more systematically the effects of electrode size by determining thresholds for pulses of 2-msec duration.

\section{Method}

In Experiment 2, the 15-mm anode was paired with cathodes 2.5, $5,10,15,20$, and $30 \mathrm{~mm}$ in diameter, whereas in Experiment 3, the 15-mm cathode was paired with anodes $2.5,5,10,15,20$, and $30 \mathrm{~mm}$ in diameter. Twelve undergraduates were paid as subjects in each experiment; 6 of the subjects participated in both experiments.

\section{Results}

In both experiments, all subjects reported sensations under the cathode on the ventral side for every pair of electrodes.

Experiment 2. The results of Experiment 2 are shown by circles in Figure 2, where mean threshold is plotted against contact area of the variable electrode (i.e., cathode). A one-way analysis of variance for repeated measures showed a significant main effect of cathode size $[F(5,55)=10.8, p<.001]$. An analysis of trend applied to the threshold data as a function of log contact area of cathode showed that the linear trend was significant $[F(1,5)=40.6, p<.01]$, accounting for $76 \%$ of the variance, and the quadratic trend was also significant $[F(1,5)$ $=12.3, p<.05]$, accounting for $23 \%$ of the variance.

Experiment 3. For comparison with the results of Experiment 2, the mean thresholds obtained in Experiment 3 are shown by the triangles in Figure 2, as a function of the contact area of the variable electrode (i.e., anode). A one-way analysis of variance for repeated measures showed a significant main effect of anode size $[F(5,55)$ 
$=30.0, p<.001]$. An analysis of trend showed that the linear trend was significant $[F(1,5)=146.8, p<.001]$, accounting for $98 \%$ of the variance. The variation due to higher order trend components was not significant.

\section{Discussion}

It appears that thresholds for the variable electrode of 15-mm diameter or less did not differ between Experiments 2 and 3, and that they could be accounted for by the significant linear trends obtained in both experiments. As is clear from Figure 2, the linear trends suggest that there was a general negative linear slope between log threshold and $\log$ area.

However, for the variable electrodes of more than $15-\mathrm{mm}$ diameter, there were threshold differences between the two experiments: The thresholds obtained in Experiment 3 decreased as anode size increased, whereas the thresholds obtained in Experiment 2 fluctuated around a constant value as a function of cathode size. The significant quadratic trend obtained in Experiment 2 may reflect this fluctuation of thresholds.

\section{EXPERIMENT 4}

In Experiment 4, we examined how the effects of electrode size on threshold interact with direction of current flow. As is shown in Figure 2, there were threshold differences between Experiments 2 and 3 for large variable electrodes. However, it was not clear whether these differences were due to the polarity of the variable electrodes or to the site of the variable electrodes.

\section{Method}

In an attempt to separate the effects of electrode site from those of electrode polarity, in Experiment 4, there were two current flows: one from the dorsal to the ventral side of the forearm, and the other from the ventral to the dorsal side of the same forearm. For each current flow, the constant electrode of $15-\mathrm{mm}$ diameter was placed on the dorsal or the ventral side, and the variable electrode of 2.5-, $15-$, or 30-mm diameter was placed on the opposite side. Fourteen undergraduates served as paid subjects.

\section{Results}

Table 1 shows frequencies of perceived locus of current ("ventral," "dorsal," and "both") as a function of site and polarity of the variable electrode. The maximal frequency for a localization category was 42 (14 subjects $\times 3$ sizes) for each condition.

Table 1

Response Frequencies of Perceived Locus of Current Pulses as a Function of Site and Polarity of the Variable Electrode

\begin{tabular}{lccccc}
\hline & \multicolumn{4}{c}{ Variable Electrode } \\
\cline { 2 - 3 } Perceived & \multicolumn{2}{c}{ Ventral } & & \multicolumn{2}{c}{ Dorsal } \\
\cline { 2 - 3 } \cline { 5 - 6 } Locus & Cathode & Anode & & Cathode & Anode \\
\hline Ventral & 41 & 20 & & 20 & 37 \\
Dorsal & 0 & 13 & & 12 & 0 \\
Both & 1 & 9 & & 10 & 5 \\
\hline
\end{tabular}

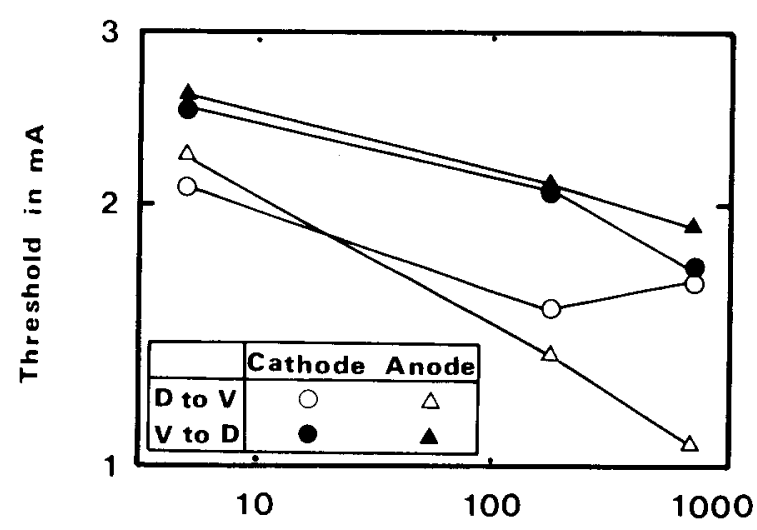

Area in $\mathrm{mm}^{2}$

Figure 3. Threshold (in milliamperes) as a function of area (in square millimeters) of variable electrode. The open symbols represent the current flow from the dorsal to the ventral; the filled symbols, the current flow from the ventral to the dorsal. The circles represent the cathodal variable electrode and the triangles represent the anodal variable electrode.

If the subjects selected loci on the basis of pure guessing, the frequency for each localization category would be an average score of 14 (i.e., 42/3) for any combination of site and polarity conditions. The $95 \%$ confidence limits derived from a binomial distribution for 14 independent trials with $p=.33$ are 1.40 and 8.3. Therefore, if the frequency is $25(8.30 \times 3)$ or more in 42 trials, it is significantly higher than chance, whereas if it is 4 $(1.40 \times 3)$ or less, it is significantly lower than chance.

Thus, the data in Table 1 suggest that when the cathode was placed on the ventral side and the anode was on the opposite side, threshold sensation was perceived mainly under the cathode, whereas when the cathode and anode were replaced by one another, the sensation was perceived under either or both electrodes.

Figure 3 shows mean threshold as a function of area of the variable electrode, with polarity and site of the variable electrode as parameters. A three-way analysis of variance for repeated measures was performed. The main effect of size was significant $[F(2,26)=30.8, p<.001]$, indicating that lower thresholds were obtained for larger variable electrodes.

The polarity $\times$ site interaction was significant $[F(1,13)$ $=60.0, p<.001]$. This suggests that lower thresholds were obtained for the cathode placed on the ventral side than for the cathode placed on the dorsal side. In other words, lower thresholds were obtained for the current flowing from the dorsal to the ventral than that for the opposite flow of current.

The site $\times$ size interaction was significant $[F(2,26)=$ $5.8, p<.01]$. This interaction is shown in the left panel of Figure 4 . The tests of simple main effects indicated that for the 2.5- and $15-\mathrm{mm}$ variable electrodes, there were no significant threshold differences between the sites of the variable electrodes, but that for the $30-\mathrm{mm}$ vari- 

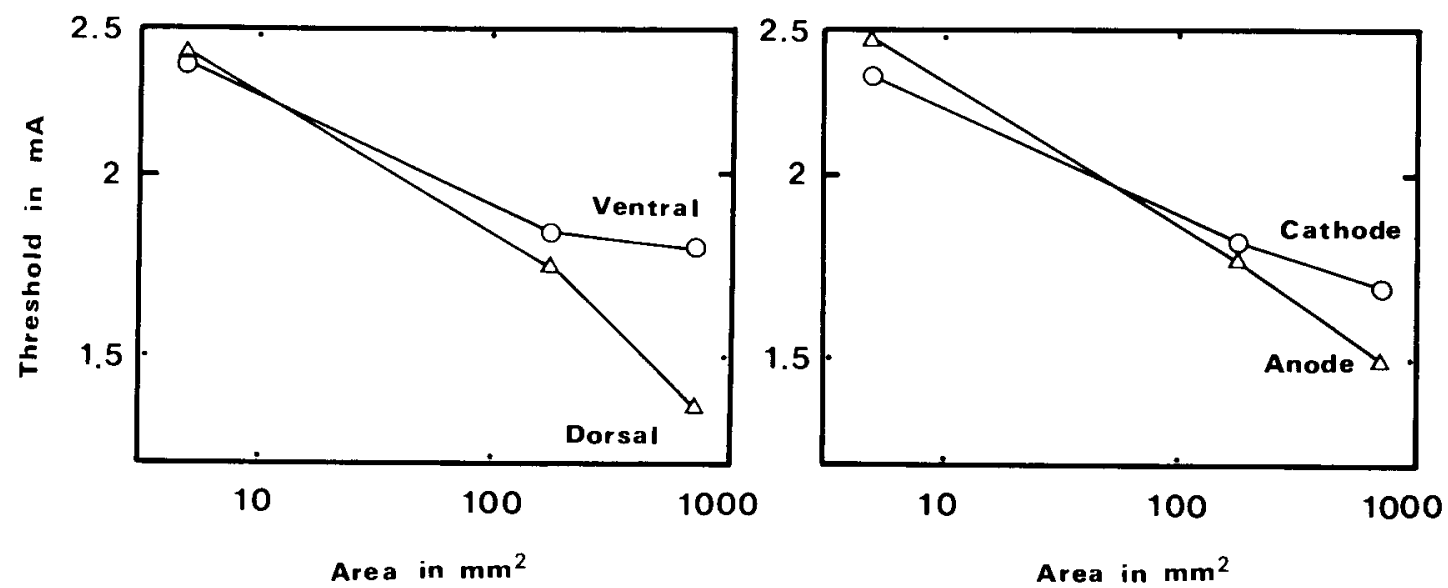

Figure 4. Threshold (in milliamperes) as a function of area (in square millimeters) of the variable electrode. Left panel: The circles represent the variable electrode on the ventral side, and the triangles represent the variable electrode on the dorsal side. Right panel: The circles represent the cathodal variable electrode, and the triangles represent the anodal variable electrode.

able electrode, the dorsal condition produced significantly lower thresholds than did the ventral condition $[F(1,39)=$ $9.8, p<.01]$.

The polarity $\times$ size interaction was significant $[F(2,26)$ $=5.9, p<.01]$. This interaction is shown in the right panel of Figure 4, which suggests that a steeper decrease of threshold was obtained with increases in electrode size for the variable anode than was obtained for the variable cathode. The tests of simple main effects showed that for the 2.5-mm-diameter electrode, the cathode condition generated significantly lower thresholds than did the anode condition $[F(1,39)=4.3, p<.05]$, but that for the 30 mm-diameter electrode, the cathode condition generated significantly higher thresholds than did the anode condition $[F(1,39)=7.1, p<.05]$.

\section{Discussion}

It is generally clear that for any combination of polarity and site of the variable electrode, threshold decreased as the size of the variable electrode increased, in agreement with the results of Experiments 1-3.

The data in Table 1 suggest that when the cathode was placed on the ventral side and the anode was on the opposite side, threshold sensation was perceived mainly under the cathode, whereas when the cathode and anode were replaced by each other, the sensation was perceived under either electrode or both electrodes. These results mean that perceived locus is determined by the flow of current, which is determined by electrode polarity and site, challenging the hypothesis that current sensation is perceived mainly under the cathode.

It is also apparent that threshold was affected by the flow of current. When current flowed from the dorsal to the ventral side, the thresholds were lower than those for the opposite flow of current. In addition, when the current flowed from the dorsal to the ventral side, the "ven- tral" localization responses amounted to $92 \%$ of total trials, whereas for the opposite flow of current, the "ventral," "dorsal," and "both" localization responses were $48 \%, 30 \%$, and $22 \%$ of total trials, respectively. This suggests that current flowing from the dorsal to the ventral side produces lower thresholds and more stable judgments of perceived locus than does current flowing from the ventral to the dorsal side.

The threshold differences between Experiments 2 and 3 may be due to the site of the variable electrode, not due to the polarity of the variable electrode. The left panel of Figure 4 shows that, when the $30-\mathrm{mm}$ variable electrode was placed on the dorsal and ventral sides, the threshold difference between the two sides amounted to $0.5 \mathrm{~mA}$, which approximated the difference obtained for the 30-mm variable electrode in Figure 2. However, the right panel of Figure 4 shows that the threshold difference between polarities for the $30-\mathrm{mm}$ variable electrode was about $0.2 \mathrm{~mA}$, which is smaller than the corresponding difference $(0.5 \mathrm{~mA})$ between Experiments 2 and 3. Moreover, the threshold difference between polarity conditions for the $2.5-\mathrm{mm}$ variable electrode was significant in Experiment 4, but not in Experiments 2 and 3.

\section{EXPERIMENT 5}

From the results of Experiments 1-4, it is clear that however current flows, threshold decreases as the size of the cathode and anode increases. Our purpose in Experiment 5 was to examine whether or not such an inverse relation between current threshold and electrode size holds true regardless of pulse duration.

\section{Method}

The $15-\mathrm{mm}$ anode was paired with the $2.5-$ or $30-\mathrm{mm}$ cathode. For each pair of electrodes, a threshold was determined for pulses 
of $1-, 10$, or $100-$ msec duration. Twelve undergraduates served as subjects.

\section{Results}

For every combination of electrode and duration, all subjects reported sensations under the cathode on the ventral side.

The thresholds obtained are shown in Figure 5, where mean threshold is plotted as a function of cathode size, with duration as the parameter.

A two-way analysis of variance for repeated measures was performed on the threshold data. The main effect of cathode size was significant $[F(1,11)=25.4, p<.001]$, indicating that the large cathode produced lower thresholds than the small one did. The main effect of duration was also significant $[F(2,22)=19.5, p<.001]$, suggesting that the pulses of shorter duration produced higher thresholds than did those of longer duration. The size $x$ duration interaction was not significant $(F=1.1)$.

Thus, it is clear that the results of Experiments 1-4 were not specific to the 2-msec duration. The dependency of threshold on electrode size was found for all pulse durations investigated.

\section{GENERAL DISCUSSION}

\section{Description of Data}

The most important finding obtained in this study is that total current threshold generally decreases as the size of the cathode and anode increases.

To describe this finding succinctly, total current thresholds obtained in Experiments 1-5 were transformed into decibel values. That is, the thresholds $I$ in each experiment were normalized to the threshold for the $30-\mathrm{mm}$ variable electrode $I_{r}$ [i.e., dB $\left.=10 \log \left(I / I_{r}\right)\right]$. These results are shown in Figure 6, where total current threshold in decibels is plotted against contact area of the variable elec-

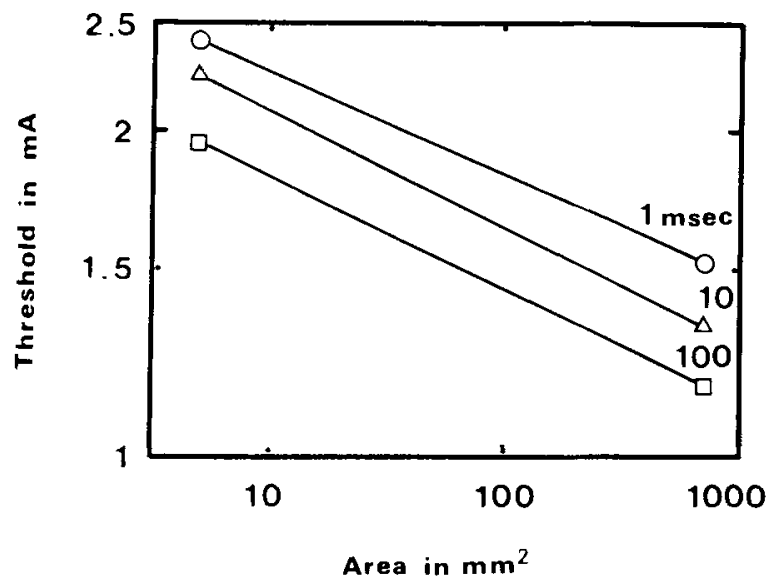

Figure 5. Threshold (in milliamperes) as a function of cathode area (in square millimeters). Current flowed from the dorsal (anode) to the ventral (cathode). The parameter is the pulse duration: circles, 1 msec; triangles, $10 \mathrm{msec}$; and squares, $100 \mathrm{msec}$. trode. The open symbols represent the conditions in which the variable cathode is on the ventral side and the $15-\mathrm{mm}$ anode is on the dorsal side; the filled symbols represent the conditions in which the variable anode is on the dorsal side and the $15-\mathrm{mm}$ cathode is on the ventral side. A smooth line was fitted by eye to each of the two electrode conditions.

Figure 6 suggests that for the variable diameters of $15 \mathrm{~mm}$ or less, there is a decrease in threshold of about $2 \mathrm{~dB}$ per every 10 -fold increase in area. To describe this current-area relation in mathematical form, the equation

$$
\left(I-I_{0}\right) A^{m}=k
$$

was fitted by least squares criteria to each of the two conditions in Figure $2 . I$ is the total current in milliamperes, $A$ is the area in square millimeters, and $I_{0}$ and $m$ are constants.

The results showed that when the variable electrode was placed on the ventral side, the value of $m$ was 0.10 $\left(I_{0}=0, \log k=0.67\right.$, and $\left.r^{2}=.74\right)$, whereas when the variable electrode was placed on the dorsal side, it was $0.20\left(I_{0}=0, \log k=0.95\right.$, and $\left.r^{2}=.98\right)$.

\section{What Is Integrated by Electrocutaneous Stimulation?}

Our results do not seem to support the current-density hypothesis, which predicts that total current threshold increases with increasing contact area of electrodes.

Likewise, our results do not seem to be in agreement with the spatial summation hypothesis, which predicts that if perfect spatial summation took place, total current threshold would remain constant, and that if partial summation or no summation took place, it would increase with increasing contact area of electrodes.

If, however, the cutaneous system integrates neural impulses discharged by current, rather than current itself passing through the skin, it seems possible to explain why total current threshold decreases as current area increases. There is evidence that when current pulses pass through the skin under an electrode, multiple conduction paths operate in parallel (Saunders, 1973). Therefore, the total current $I$ equals the sum of currents passing along all conduction paths:

$$
I=\sum I_{i}
$$

where $I_{i}$ is a current passing the $i$ th path $(i=1, \ldots n)$ and $\sum$ is an abbreviation for $\sum_{i=1}^{n}$. According to Saunders's data, the conduction paths are distributed equally under the electrode, at the rate of a path per square millimeter. It follows that the number of conduction paths increases as the contact area of electrodes increases.

We assume here that the cutaneous system sums up neural impulses discharged by the nerves near these conduction paths, and that a threshold sensation arises when the added impulses reach a particular value, $E$. Thus, we have

$$
E=\Sigma e_{i},
$$


where $e_{i}$ is the number of impulses per unit time (i.e., the rate of neural firing), generated at the $i$ th conduction path.

Furthermore, if the rate of neural firing at each conduction path is proportional to the logarithm of stimulus current, we have

$$
e_{i}=K \log I_{i}
$$

where $K$ is constant.

From Equations 3 and 4, we obtain

$$
E=K \sum \log l_{i} \text {. }
$$

When $l_{i}=I / n$, Equation 5 is transformed:

$$
E=K \sum \log \frac{I}{n}=K n \log \frac{I}{n} .
$$

Hence, we have

$$
\log I=\log n+\frac{E}{K n} .
$$

Figure 7 shows $\log I$ in arbitrary units as a function of $\log n$, for the $E / K$ values of $10,20,30$, and 40 . Note that the right hand of Equation 7 is a simple sum of the two terms: The first term, $\log n$, increases as a function of $n$, whereas the second term, $E /(K n)$, decreases as a function of $n$. This means that $\log I$ is a $U$-shaped function of $\log n$.
Since the number of conduction paths is proportional to the contact area of electrodes, it is clear from Figure 7 that as the contact area of the electrodes increases, the threshold decreases rapidly, then flattens, and finally increases slightly. It seems that our data are in agreement with the general trends predicted by the neural summation model.

\section{Effects of Electrode Polarity and Site on Threshold}

Experiment 4 suggested that current flowing from the dorsal to the ventral side produces lower thresholds than the opposite flow of current does. This may be explained by assuming that the cathode is more likely to produce neural depolarization than the anode is, and that the ventral skin is more sensitive to current than the dorsal skin is. The first assumption can be supported with reference to the study of monopolar stimulation by Gibson (1968), who found that cathodal threshold is one half to three fourths the anodal threshold. Furthermore, BeMent and Ranck (1969) and Ranck (1981) suggested that the depolarization of nerves, by which action currents flow in nerves, takes place more easily under the cathode than under the anode.

For the second assumption, there may be no direct evidence, but some research has shown that the sensitivity to current varies according to the body site stimulated. For example, Bujas, Szabo, Kovacic, and Rohacek (1975)

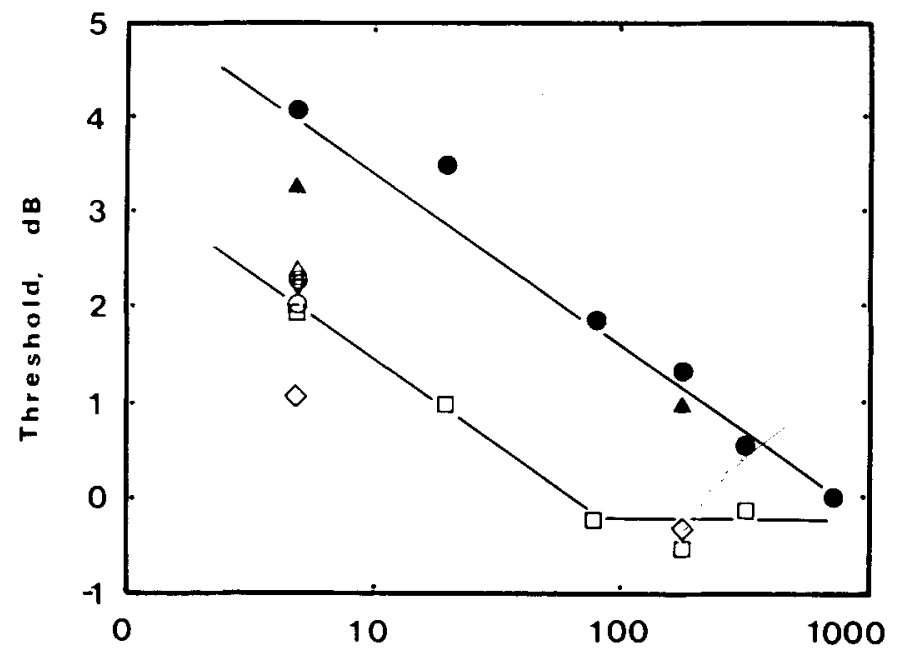

Area in $\mathrm{mm}^{2}$

Figure 6. Threshold (in decibels) as a function of area (in square millimeters) of variable electrode. Current flowed from the dorsal (anode) to the ventral (cathode). The filled symbols represent the conditions in which the $15-\mathrm{mm}$ cathode is on the ventral side and the variable anode is on the dorsal side. : Experiment 3. $\Delta$ : Experiment 4. The open symbols represent the conditions in which the variable cathode is on the ventral side and the 15-mm anode is on the dorsal side. (): Experiment 1. $\square$ : Experiment 2. $\bigcirc$ : Experiment 4. $O$ : the 1-msec condition in Experiment 5. $\nabla:$ the 10-msec condition in Experiment 5. $\triangle$ : the 100-msec condition in Experiment 5. 


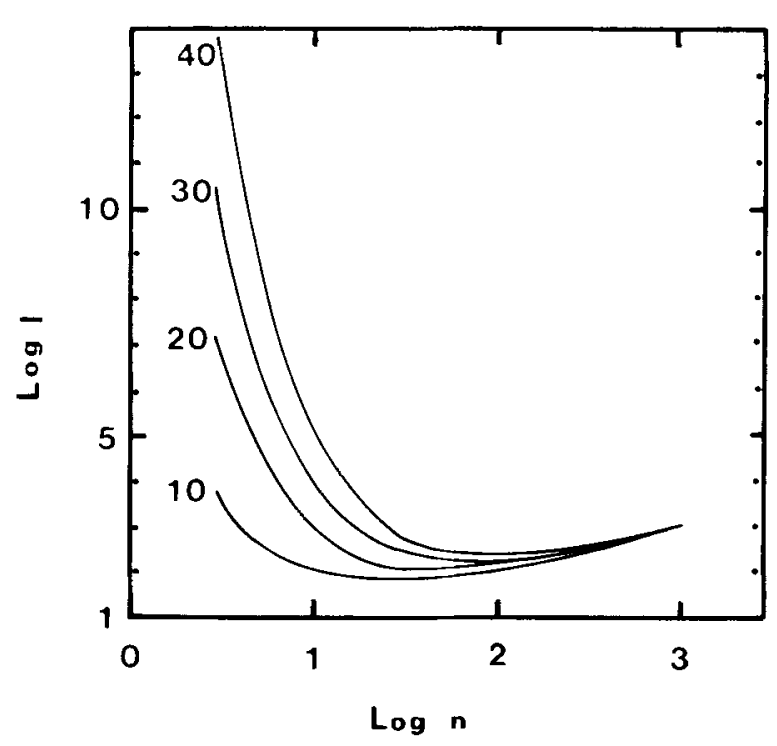

Figure 7. Log threshold in arbitrary units as a function of $\log$ conduction paths. The parameter is the value of $E / K$.

compared thresholds of the tongue, eye, and finger for current, and found that the tongue is the most sensitive, the finger is the least sensitive, and the eye is intermediate.

\section{Effects of Electrode Polarity and Site on the Perceived Locus}

Table 1 suggests that when current flows from the dorsal (anode) to the ventral (cathode) side, the threshold sensation is perceived mainly under the cathode, but that in the opposite flow of current, the perceived locus was not consistent among the subjects.

Therefore, the perceived locus may also be determined both by electrode polarity and by body site stimulated. For the current flowing from the dorsal to the ventral side, the neural depolarization would occur mostly under the cathode on the ventral side, because the sensitivity expected from electrode polarity coincides with that expected from body site. However, the perception of locus is not so simple with the opposite flow of current. If the subjects based their judgments on the sensitivity of the body site that was stimulated, the sensations would be perceived on the ventral side; if the subjects based their judgments on electrode polarity, the sensation would be perceived on the dorsal side. We think that the discrepancy between the two types of sensitivity causes the instability in perceived locus when the current is flowing from the ventral to the dorsal side.

There are other possible ways to explain the perceived locus. Békésy (1959a) showed that when two vibrators were each placed on two fingers, the sensation was localized at one site-usually somewhere between the fingers. His observation is surely true in some circumstances, but our subjects did not perceive sensations at a deep site of the forearm; they perceived it on the skin surface under either electrode or both of them.
Békésy (1963) also said that when two different sites were stimulated, the sensation was localized at the more proximal site. For example, when the finger touches the cheek, we are likely to perceive the face to be touched, but when the finger touches the leg, we are likely to perceive the finger to be touched. However, this explanation is not applicable to our results, because the ventral and dorsal sites have nearly the same proximity of stimulation.

\section{Effect of Electrode Configuration on Threshold}

The finding that threshold generally decreases with contact area of electrodes does not seem to agree with our previous study (Higashiyama \& Tashiro, 1983), which showed that when two electrodes were placed side by side on the ventral side of the forearm, threshold is determined by the lateral separation of electrodes, rather than by the area of the electrodes.

This discrepancy between our two studies may be due to the difference in electrode configuration. In the present study, current penetrated the deep tissue of the forearm, but in the side-by-side electrodes used in the previous study, a local short circuit was probably created by the electrodes' coming into contact with each other, and, as a result, much current flowed along the skin surface.

Several electrode configurations have been used in electrocutaneous stimulation. Each configuration has both merits and demerits for the examination of the effects of electrode size on electrocutaneous sensation. One typical configuration is what Gibson (1968) called a monopolar configuration, in which a very large ("indifferent") electrode is placed under the sole of the foot, and a smaller ("active") electrode is placed at the sites where the stimulation is to be located. In the monopolar configuration, two electrodes do not yield interactions such as short circuits or collision blocks (van den Honert \& Mortimer, 1981a, 1981b), but this arrangement produces stray current paths through the body and might give unwanted effects (Sherrick \& Cholewiak, 1986). On the other hand, Békésy (1959b) used concentric electrodes separated by several millimeters. The concentric configuration can limit current flow in the tissue to the surface just below electrodes, but it is likely to generate a leak between them.

\section{REFERENCES}

BÉKÉSY, G. voN (1959a). Neural funneling along the skin and between the inner and outer hair cells of the cochlea. Journal of the Acoustical Society of America, 31, 1236-1249.

BÉKÉSY, G. VON (1959b). Synchronism of neural discharges and their demultiplication in pitch perception on the skin and in hearing. Journal of the Acoustical Society of America, 31, 338-349.

BÉKÉSY, G. VON (1963). Interaction of paired sensory stimuli and conduction in peripheral nerves. Journal of Applied Physiology, 18, 1276-1284.

BEMENT, S. L., \& RANCK, J. B., JR. (1969). A quantitative study of electrical stimulation of central myelinated fibers. Experimental Neurology, 24, 147-170.

Benton, L. A., Baker, L. L., Bowman, B. R., \& Waters, R. L. (1981). Functional electrical stimulation: Practical clinical guide. Downey, CA: Rancho Los Amigos Rehabilitation Engineering Center. Bujas, Z., Szabo, S., Kovacic, M., \& RohaceK, A. (1975). Sensory 
scales for electrical stimuli in three sense modalities. Acta Instituti Psychologici, 75, 17-23.

GrBsoN, R. H. (1968). Electrical stimulation of pain and touch. In D. R. Kenshalo (Ed.), The skin senses (pp. 223-261). Springfield, IL: Thomas.

GreEn, R. T. (1962). The absolute threshold of electrical shock. British Journal of Psychology, 52, 107-115.

Higashiy ama, A., \& Tashiro, T. (1983). Temporal and spatial integration for electrocutaneous stimulation. Perception \& Psychophysics, $33,437-442$.

Higashiyama, A., \& Tashiro, T. (1988). Temporal integration of double electrical pulses. Perception \& Psychophysics, 43, 172-178.

Kenshalo, D. R. (1970). Psychophysical studies of temperature sensitivity. In W. D. Neff (Ed.), Contributions to sensory physiology (Vol. 4, pp. 19-74). New York: Academic Press.

MARKS, L. E. (1974). Sensory processes: The new psychophysics. New York: Academic Press.

McNeal, D. R. (1973). Peripheral nerve stimulation-Superficial and implanted. In W. S. Field \& L. A. Leavitt (Eds.), Neural organization (pp. 77-99). New York: Intercontinental Medical Book Corp.

RANCK, J. B., JR. (1981). Extracellular stimulation. In M. M. Patterson \& R. P. Kesner (Eds.), Electrical stimulation research techniques (pp. 1-36). New York: Academic Press.

Rosenthal, R., \& Rosnow, R. L. (1985). Contrast analysis: Focused comparisons on the analysis of variance. London: Cambridge University Press.
SAUNDERS, F. A. (1973). Electrocutaneous displays. In F. A. Geldard (Ed.), Cutaneous communication systems and devices (pp. 20-26). Austin, TX: Psychonomic Society.

Sherrick, C. E., \& Cholewiak, R. W. (1986). Cutaneous sensitivity. In K. R. Boff, L. Kaufman, \& J. P. Thomas (Eds.), Handbook of perception and human performance: Vol. I. Sensory processes and perception (chap. 12). New York: Wiley.

TURSky, B., \& WATSON, P. D. (1964). Controlled physical and subjective intensities of electrical shock. Psychophysiology, 1, 151-162.

VAN DEN HONERT, C., \& MORTIMER, J. T. (1981a). A technique for collision block of peripheral nerve: Frequency dependence. IEEE Transactions of Biomedical Engineering, BME-28, 379-382.

VAN DEn Honert, C., \& Mortimer, J. T. (1981b). A technique for collision block of peripheral nerve: Single stimulus analysis. IEEE Transactions of Biomedical Engineering, BME-28, 373-378.

Vernon, J. A., \& WeSSMan, A. (1956). The effect of phase manipulation upon electrocutaneous stimulation. Journal of Comparative \& Physiological Psychology, 49, 293-296.

Verrillo, R. T. (1963). Effect of contactor area on the vibrotactile threshold. Joumal of the Acoustical Society of America, 35, 1962-1966.

(Manuscript received July 13, 1989; revision accepted for publication May 24, 1990.) 